\title{
DUAL BIOLOGICAL THERAPY IN A PATIENT WITH CROHN'S DISEASE AND NONRADIOGRAPHIC AXIAL SPONDYLOARTHRITIS: A CASE REPORT
}

Pedro Laurindo Fiorio Júnior ${ }^{1}$, Ana Cláudia Guimarães Carvalho' ${ }^{1}$, Victória Dornelas Paz Carvalho ${ }^{1}$, Maria Cecília Dias Corrêa ${ }^{1}$, Aline Teixeira de Landa ${ }^{1, *}$, Renata Henriques de Azevedo ${ }^{1}$, Viviane Angelina de Souza ${ }^{1}$, Louise Fellet Barbosa ${ }^{1}$

1.Universidade Federal de Juiz de Fora, Juiz de Fora (MG), Brazil.

*Corresponding author: aalineland@yahoo.com.br

\section{BACKGROUND}

In the present study we report a case of a 49-year-old male patient, followed by the Rheumatology service of the Hospital Universitário da Universidade Federal de Juiz de Fora, with nonradiographic axial spondyloarthritis associated with inflammatory bowel disease in need of double biological therapy to control rheumatological and gastroenterological diseases. This report is justified by the scarcity of similar descriptions in the literature.

\section{CASE REPORT}

A 49-year-old male patient, rural worker, with leukoderma, resident in Rio Novo, a city located in the state of Minas Gerais. Diagnosis of Crohn's disease in 2018, inflammatory phenotype (ileocolitis). Initial treatment with azathioprine began in September 2018 with replacement in February 2019 to ustekinumab due to the maintenance of disease activity, obtaining satisfactory control. In March 2019, he began his follow-up with the rheumatology team of the Hospital Universitário da Universidade Federal de Juiz de Fora, due to inflammatory low back pain and morning stiffness ( $>1 \mathrm{~h}$ ); associated with alterations compatible with sacroiliitis in magnetic resonance imaging, without radiographic alterations, HLA-B27 negative. Diagnosis of nonradiographic axial spondyloarthritis associated with inflammatory bowel disease was performed. Since the patient had already started using ustekinumab, obtaining Crohn's disease good control, and presented contraindication to the use of anti-inflammatory drugs, he chose to maintain such therapy and observe the clinical response. Patient maintained satisfactory evolution for both conditions until December 2020, when low back pain was considered inflammatory, presenting BASDAI of 5.4. For the good control of inflammatory bowel disease and axial disease, we opted in a joint decision with the gastroenterology team, for the maintenance of ustekinumab in association with adalimumab. The patient then evolved with good control of both conditions, presenting BASDAI below 4 in all subsequent consultations, indicating mild or inactive disease.

\section{CONCLUSION}

Although there is no recommendation for double biological therapy for the treatment of axial spondyloarthritis associated with inflammatory bowel disease, there are reports of this association for the treatment of Crohn's disease. In the present case, the association of ustekinumab with adalimumab was effective for controlling intestinal and axial manifestations, with no adverse effects to date. Dual biological therapy may be a treatment option for patients with axial spondyloarthritis associated with inflammatory bowel disease, since they have contraindication for standard treatment with non-steroidal anti-inflammatory drugs and anti-interleukin 17.

\section{KEYWORDS}

Crohn's disease, Spondyloarthritis, Dual biological therapy. 